Mev. In comparing the relative transition probabilities it must be remembered that the pairs are approximately twenty times as effective in producing ionization as are the gamma-rays, hence the latter are in all cases much more numerous.

It is interesting that although these pairs occur at definite resonance energies they do not coincide with the gamma-ray resonances. For example, at $920 \mathrm{kv}$ the ionization due to gamma-radiation is nearly ten times as intense as that produced by pairs, while at 840 and $1190 \mathrm{kv}$ the reverse is true. Clearly, therefore, the pairs are not produced by internal conversion of gamma-rays in the usual sense, but are emitted directly from a nucleus in an excited state. Presumably this is a state in $\mathrm{O}^{16}$ with $j=0$ and the transition is to the ground state which is known to have $j=0$. It is, however, possible that some or all of the pairs observed originate from a transition in $\mathrm{Ne}^{20}$. If so, gammarays of approximately $7.5 \mathrm{Mev}$ energy should accompany the pairs and we cannot exclude this possibility from our experiments so far. If the transition is in $\mathrm{O}^{16}$ alpha-particles should be observable at the pair resonances. Since no single quantum transition is allowed from such a state, the lifetime must be very long. Professor Oppenheimer has made a tentative estimate of $10^{-4}$ second for the halflife.

It seems probable that the pairs observed by Crane and Halpern $^{8}$ at $600 \mathrm{kv}-11$ pairs per thousand quanta-are to be attributed to this process rather than to internal conversion.

W. A. FOWLER

California Institute of Technology,

C. C. Lauritsen

Pasadena, California,
September 27, 1939.

1 L. R. Hafstad and M. A. Tuve, Phys. Rev. 48, 306 (1935).

2 Bernet, Herb and Parkinson, Phys. Rev. 54, 398 (1938).

3 Dee, Curran and Strothers, Nature 143, 759 (1939)

${ }^{4}$ Fowler, Lauritsen and Lauritsen, Bulletin Phys. Soc. Stanford

Meeting, June (1939)

5 McLean, Becker, Fowler and Lauritsen, Phys. Rev. 55, 796 (1939).

${ }^{6} \mathrm{~W}$. E. Burcham and C. L. Smith, Nature 143, 795 (1939).

${ }^{7}$ We are indebted to Dr. Burcham for communicating these results

to us before publication.

\section{Low Energy Gamma-Radiation from Lithium Bombarded with Protons}

By means of the method described in the preceding letter we have investigated the radiation from $\mathrm{Li}^{7}+\mathrm{H}^{1}$ as function of proton energy. The target used consisted of separated $\mathrm{Li}^{7}$ with a stopping power of approximately $30 \mathrm{kev} .^{1}$

The filter used in front of ionization chamber No. II was $1.33 \mathrm{~cm}$ of lead. The readings of chambers Nos. I and II are shown in curves 1 and 2, respectively, and curve 3 is the difference between 1 and 2 (Fig. 1).

The resonance at $440 \mathrm{kev}$ appears to be due entirely to the well-known $17.5 \mathrm{Mev}$ gamma-radiation, while the radiation above $800 \mathrm{kev}$ is strongly absorbed in lead.

We have measured the attenuation of the radiation at 1080 and $1290 \mathrm{kev}$ and find an apparent absorption coefficient $\mu=1.50 \pm 0.10 \mathrm{~cm}^{-1}$ in lead and $\mu=0.12 \pm 0.01 \mathrm{~cm}^{-1}$ in aluminum. For annihilation radiation from $\mathrm{N}^{13}$ we find,

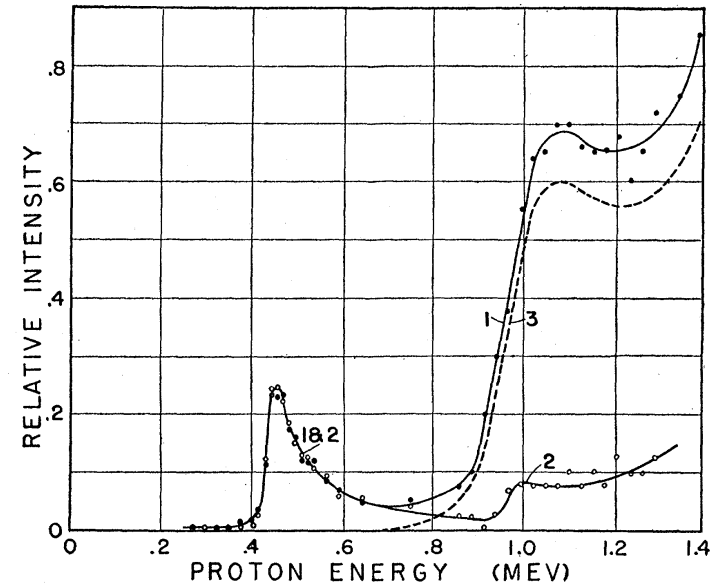

FIG. 1. Relative intensity of ionization vs. bombarding energy Curve 1 -radiation filtered by $0.15 \mathrm{~mm}$ lead. Curve 2 -radiation filtered

with the same arrangement, $\mu=1.43 \pm 0.05 \mathrm{~cm}^{-1}$ in lead. This gives the value $495 \pm 25 \mathrm{kev}$ for the gamma-rays at 1080 and $1290 \mathrm{kev}$ bombarding energy.

From this and from the character of the yield curve above $800 \mathrm{kev}$ it seems reasonable to assume that this radiation originates from excitation of $\mathrm{Li}^{7}$ without capture of the proton in analogy with the well-known excitation of $\mathrm{Li}^{7}$ by $\mathrm{He}^{4}$. Rumbaugh, Roberts and Hafstad ${ }^{2}$ have observed the same state in the following reactions:

$$
\begin{aligned}
\mathrm{Li}^{6}+\mathrm{H}^{2} \rightarrow\left(\mathrm{Be}^{8}\right) & \rightarrow \mathrm{Li}^{7}+\mathrm{H}^{1} \\
& \rightarrow * \mathrm{Li}^{7}+\mathrm{H}^{1} \\
& \rightarrow \mathrm{Be}^{7}+n^{1} \\
\mathrm{Be}^{7} & \rightarrow * \mathrm{Li}^{7}-e^{-} .
\end{aligned}
$$

From the difference in proton ranges they find the separation of the states in $\mathrm{Li}^{7}$ to be $455 \pm 15 \mathrm{kev}$, and from the attenuation of the radiation following the decay of $\mathrm{Be}^{7}$, $425 \pm 25 \mathrm{kev}$.

Although our value $495 \pm 25 \mathrm{kev}$ is somewhat higher there can be little doubt that the same state in $\mathrm{Li}^{7}$ is involved.

$$
\begin{aligned}
& \text { W. A. FOWLER } \\
& \text { C. C. Lauritsen }
\end{aligned}
$$

\section{On the Equality of the Proton-Proton and Proton-Neutron Interactions}

A comparison of the ${ }^{1} S$ proton-proton interaction and the ${ }^{1} S$ proton-neutron interaction has been made recently by Breit, Hoisington, Share and Thaxton..$^{1}$ It is the purpose of this letter to add a remark to this subject. With the meson type of potential, $C e^{-\lambda r} / r \lambda$, a variational calculation has been made of the binding energy of $\mathrm{H}^{3}$ of high accuracy (error $<0.1$ percent). ${ }^{2}$ This calculation together with the value of the binding energy of $\mathrm{H}^{2}$ and the scattering of 